\title{
Factores predictivos de cardiotoxicidad inducida por antraciclinas en una cohorte retroprolectiva de niños supervivientes de cáncer
}

\author{
Rodolfo Rivas-Ruiz, ${ }^{1}$ Kingston Ureña-Wong, ${ }^{2 *}$ Osvaldo Daniel Castelán-Martínez, ${ }^{3}$ \\ Yadira Betanzos-Cabrera, ${ }^{4}$ César Lazo-Cárdenas, ${ }^{4}$ César Ramírez-Portillo ${ }^{4}$ y Enrique López-Aguilar ${ }^{4}$ \\ ${ }^{1}$ Instituto Mexicano del Seguro Social, Centro de Adiestramiento e Investigación Clínica, Coordinación de Investigación en Salud; ${ }^{2}$ Asociación para \\ Evitar la Ceguera en México, I. A. P.; ${ }^{3}$ Universidad Nacional Autónoma de México, Facultad de Estudios Superiores Zaragoza, Laboratorio de \\ Farmacología Clínica; "Instituto Mexicano del Seguro Social, Centro Médico Nacional Siglo XXI, Hospital de Pediatría "Dr. Frenk Freund". Ciudad \\ de México, México
}

\section{Resumen}

Introducción: La cardiotoxicidad es una reacción adversa asociada al uso de antraciclinas. Objetivo: Estimar los factores asociados al desarrollo de cardiotoxicidad por antraciclinas en pacientes pediátricos supervivientes de cáncer. Método: Cohorte retroprolectiva de niños con diagnóstico de cáncer tratados con antraciclinas. Se realizó determinación ecocardiográfica basal de la fracción de expulsión (FEViO) antes del inicio del tratamiento y a los 12 meses (FEVi1). Del expediente se obtuvieron las características demográficas y el tratamiento. Se realizó un modelo de regresión logística múltiple (RLM); la FEVi1 < 50 \% fue la variable dependiente, que se ajustó por las principales variables confusoras. Resultados: Se incluyeron 65 pacientes, 36.9 \% fue del sexo femenino y 56.8 \% presentó un tumor sólido. La FEViO fue de $74.79 \pm 7.3$ \% y la FEVi1, de $67.96 \pm 6.7 \%(p=0.001) ; 60 \%$ desarrolló cardiotoxicidad. En la RLM solo la dosis acumulada $>430 \mathrm{mg}$ se asoció a cardiotoxicidad $(p=0.001)$. Conclusiones: En los niños mexicanos se debe evitar una dosis acumulada $>430 \mathrm{mg}$ de antraciclinas para evitar la cardiotoxicidad.

PALABRAS CLAVE: Antraciclinas. Cardiotoxicidad. Factores de riesgo.

\section{Predictors of anthracycline-induced cardiotoxicity in a retro-prolective cohort of children surviving cancer}

\begin{abstract}
Introduction: Cardiotoxicity is an adverse reaction associated with the use of anthracyclines. Objective: To estimate the factors associated with the development of anthracycline cardiotoxicity in pediatric patients surviving cancer. Method: Retro-prolective cohort of children diagnosed with cancer and treated with anthracyclines. Baseline echocardiographic determination of ejection fraction (LVEFO) was carried out before the start of treatment and again at 12 months (LVEF1). Demographic characteristics and treatment were obtained from the medical record. A multiple logistic regression (MLR) model was constructed; LVEF1 $<50 \%$ was the dependent variable, which was adjusted for the main confounding variables. Results: Sixty-five patients were included, out of which $36.9 \%$ were females and $56.8 \%$ had a solid tumor. LVEFO was $74.79 \pm 7.3 \%$ and LVEF1, $67.96 \pm 6.7 \%(p=0.001) ; 60 \%$ developed cardiotoxicity. In the MLR, only a cumulative dose $>430 \mathrm{mg}$ was associated with cardiotoxicity $(p=0.001)$. Conclusions: In Mexican children, an anthracycline cumulative dose $>430 \mathrm{mg}$ should be avoided in order to prevent cardiotoxicity.
\end{abstract}

KEY WORDS: Anthracyclines. Cardiotoxicity. Risk factors.

Correspondencia:

*Kingston Ureña-Wong

E-mail: kingston.uw94@gmail.com
Gac Med Mex. 2020;156:218-224

Disponible en PubMed

www.gacetamedicademexico.com CC BY-NC-ND (http://creativecommons.org/licenses/by-nc-nd/4.0/). 


\section{Introducción}

La incidencia del cáncer en México se ha estimado en 126 casos/millón/año. ${ }^{1,2}$ Informes realizados por la Organización Mundial de la Salud demuestran que la incidencia de cáncer en menores de 15 años ha aumentado en más de $200 \%$ en los países en desarrollo, con predominio de las leucemias y los linfomas. ${ }^{3-5}$ Además del incremento de la incidencia mundial de cáncer, los tratamientos han mejorado, logrando incrementar la supervivencia, pero también se han incrementado las reacciones adversas asociadas a los tratamientos. ${ }^{6}$ Respecto a las antraciclinas, ${ }^{7}$ se han reportado eventos adversos, tanto durante la administración de la terapia como a largo plazo.

Los pacientes pediátricos sobrevivientes de cáncer tienen ocho veces mayor probabilidad de morir por enfermedades cardiacas y 15 veces mayor probabilidad de sufrir insuficiencia cardiaca que sus hermanos que no tuvieron cáncer. ${ }^{8,9}$ La cardiotoxicidad (CTX) es la principal causa no cancerosa de complicaciones graves y el riesgo persiste hasta 45 años después de finalizar el tratamiento; además de constituir la segunda causa de muerte, después del cáncer secundario. ${ }^{10}$

Los pacientes sobrevivientes de cáncer infantil tratados con antraciclinas cursan con disfunción cardiaca subclínica hasta seis años después de completar el tratamiento. ${ }^{1}$ Asimismo, la CTX se puede desarrollar a largo plazo y ocurrir entre los cuatro y 20 años después de finalizar el tratamiento. ${ }^{11}$ Algunos estudios reportan que la prevalencia de esta complicación llega a $57 \% .^{12}$ En México no se conoce la incidencia de CTX en los niños supervivientes de cáncer.

La CTX aguda se puede manifestar desde cambios leves en el electrocardiograma hasta arritmias potencialmente mortales. ${ }^{13}$ Se ha descrito que los efectos cardiotóxicos a largo plazo dependen de las dosis de los fármacos ${ }^{14}$ y son clínicamente más importantes que los agudos.

Si bien se han descrito algunos factores asociados a la CTX aguda, pocos estudios se abocan a los factores que se asocian al desarrollo de CTX a largo plazo. El objetivo del presente estudio fue evaluar los factores asociados al desarrollo de CTX a largo plazo en niños supervivientes de cáncer tratados con antraciclinas.

\section{Método}

Estudio de cohorte retroprolectiva de pacientes sobrevivientes de cáncer atendidos en el Servicio de
Oncología del Hospital de Pediatría "Dr. Silvestre Frenk Freund" entre 2013 y 2018. Se incluyeron pacientes < 16 años sobrevivientes de cáncer (periodo libre de enfermedad $\geq 5$ años), que recibieron tratamiento con antraciclinas, sin evidencia de enfermedad cardiaca previa corroborada por ecocardiografía. Todos los participantes debían contar con un ecocardiograma basal obtenido en el primer mes de iniciado el tratamiento con antraciclinas, en el Servicio de Cardiología por los médicos de base. Se excluyeron los pacientes con anemia clínica, corroborada por biometría hemática (hemoglobina $<7 \mathrm{mg} / \mathrm{dL}$ ), o con repercusión hemodinámica. En el Servicio de Cardiología Pediátrica del hospital se realizó una determinación de fracción de expulsión antes del tratamiento (FEVi0) y a los 12 meses de iniciado el mismo (FEVi1). Del expediente se obtuvo la información de las características demográficas y del tratamiento. Se definió como CTX a la disminución de $10 \%$ de la FEVi1 respecto al valor basal, conforme a las guías para tratamientos contra el cáncer y toxicidad cardiovascular de la Sociedad Europea de Cardiología..$^{15}$

Se calculó el tamaño de la muestra con la fórmula de diferencia de proporciones, asumiendo un alfa $=0.05$ y 1 -beta $=0.20$. Se utilizó el programa Epi Info. Se estimó $8 \%$ de sujetos con CTX anterior a la quimioterapia y $40 \%$ a un año del tratamiento. La muestra mínima quedó definida en 60 pacientes, tamaño que aseguró la estabilidad de los modelos de regresión logística múltiple (RLM) ajustados por seis variables.

En la estadística descriptiva se resumieron las siguientes variables cuantitativas: edad, peso, talla, FEViO, FEVi1. Para corroborar el tipo de distribución se llevaron a cabo métodos inferenciales; si las variables no mostraban distribución normal con este procedimiento, se realizaron pruebas de normalidad de Kolmogórov-Smirnoff. Las variables que tuvieron distribución normal fueron registradas con media y desviación estándar y las variables de libre distribución, con mediana y rangos intercuartílicos 25-75 (RIC). Las variables nominales (sexo, edad dicotómica, tipo de tumor) se registraron con frecuencias y porcentajes.

Para determinar los factores de riesgo se analizaron las variables nominales mediante $\chi^{2}$ de Pearson. Para evaluar la relevancia clínica se calcularon riesgos relativos (RR) con intervalo de confianza de $95 \%$ (IC $95 \%$ ). Las variables cuantitativas con distribución normal se analizaron con t de Student o $U$ de MannWhitney. Las variables que resultaron estadísticamente 
significativas en el modelo bivariado fueron seleccionadas para ser incluidas en el modelo de RLM, el cual se realizó por pasos descendentes, con el objetivo de encontrar las variables independientes de mayor asociación. Para evaluar la dosis de mayor riesgo para CTX se realizó una curva $\mathrm{ROC}$, a fin de encontrar el punto de mayor razón de verosimilitud positiva (RVP).

Para el análisis estadístico se utilizó el programa SPSS versión 25. Un valor de $p<0.05$ se determinó como estadísticamente significativo en todos los casos.

El presente estudio se realizó siguiendo los lineamientos éticos de la investigación en seres humanos ${ }^{16}$ indicados en la Declaración de Helsinki, el Código de Núremberg, el Informe Belmont, las Normas CIOMS y las Buenas Prácticas Clínicas para las Américas, así como de acuerdo con el Reglamento de la Ley General de Salud en Materia de Investigación para la Salud, en particular el artículo 13 concerniente al respeto, dignidad y protección de los derechos del paciente pediátrico; el artículo 17 , respecto al cual se consideró una investigación de riesgo mínimo; y el 20 y 21 , que regulan el consentimiento y asentimiento informados. La investigación también se circunscribió a las indicaciones de la Norma oficial mexicana NOM 012-SS3-2012 para la ejecución de proyectos de investigación para la salud en seres humanos. Se invitó a participar a todos los pacientes que recibieron antraciclinas y que se encontraban en la cohorte de supervivientes de cáncer (selección equitativa de los sujetos). Todos los padres de los pacientes que aceptaron participar en el protocolo firmaron una carta de consentimiento previa información de los objetivos de la investigación. Los niños mayores de ocho años firmaron cartas de asentimiento informado.

Como parte del protocolo de seguimiento, todos los pacientes recibieron dexrazoxano como cardioprotector. Aquellos en quienes se detectó CTX fueron enviados al Servicio de Cardiología para recibir un esquema de inhibidores de la enzima convertidora de angiotensina y se les hizo seguimiento (balance de riesgo-beneficio).

El estudio tiene valor social debido a que en la actualidad los supervivientes de cáncer no son evaluados de modo sistemático en la práctica pediátrica. Se contactará a los padres de los participantes para informarles de los resultados de la investigación.

La investigación fue revisada y aceptada por la Comisión Nacional de Investigación Científica del Instituto Mexicano del Seguro Social con el número R-2013-785-040.
Tabla 1. Características basales de 65 niños supervivientes de cáncer

\begin{tabular}{|c|c|c|}
\hline Variable & $n$ & $\%$ \\
\hline $\begin{array}{l}\text { Sexo } \\
\text { Femenino }\end{array}$ & 24 & 36.9 \\
\hline $\begin{array}{l}\text { Edad al diagnóstico } \\
\quad \leq 5 \text { años } \\
>5 \text { años }\end{array}$ & $\begin{array}{l}26 \\
39\end{array}$ & $\begin{array}{l}40.0 \\
52.3\end{array}$ \\
\hline $\begin{array}{l}\text { Tipo de tumor } \\
\text { Hematolinfoide } \\
\text { Sólido }\end{array}$ & $\begin{array}{l}26 \\
39\end{array}$ & $\begin{array}{l}40.0 \\
60.0\end{array}$ \\
\hline Peso inicial en kg (RIC) & \multicolumn{2}{|c|}{$15.5(11.5-24.7)$} \\
\hline Dosis acumulada en $\mathrm{mg}(\mathrm{RIC})^{\star}$ & \multicolumn{2}{|c|}{$320(165-485)^{\star}$} \\
\hline Talla inicial en cm & \multicolumn{2}{|c|}{$107 \pm 27$} \\
\hline FEViO en \% (media) & \multicolumn{2}{|c|}{$74.79 \pm 7.3$} \\
\hline FEVi1 en \% (media) & \multicolumn{2}{|c|}{$67.96 \pm 6.7$} \\
\hline
\end{tabular}

\section{Resultados}

Se reclutaron 65 pacientes que cumplieron con los criterios de inclusión. Las características basales de la población se resumen en la Tabla 1. El $36.9 \%$ de los participantes fue del sexo femenino. En cuanto a la edad, $60 \%$ de los participantes fue mayor de cinco años al momento del diagnóstico. Las neoplasias sólidas representaron $56.8 \%$ de los tumores. El peso basal de los participantes fue de $15.5 \mathrm{~kg}(\mathrm{RIC}=11.5-24.7 \mathrm{~kg})$, la talla inicial fue de $107 \pm 26.6 \mathrm{~cm}$. La dosis acumulada de antraciclinas (epirrubicina, doxorrubicina o daunorrubicina) tuvo una mediana de $320 \mathrm{mg} / \mathrm{m}^{2}$ de superficie corporal (RIC = 165-485). La FEVi0 de los participantes tuvo una media de $74.8 \pm 7.3 \%$.

Treinta y nueve pacientes (60\%) desarrollaron CTX temprana. Al analizar los factores de riesgo encontramos que el sexo masculino tuvo un $\mathrm{RR}=2.09$ (IC $95 \%=0.7-6.13$ ) para desarrollo de cardiotoxicidad. De los tres tipos de antraciclinas utilizadas en el estudio, la epirrubicina presentó un $\mathrm{RR}=2.35$ (IC $95 \%=0.7-7.61)$. El tipo de tumor hematolinfoide presentó un $\mathrm{RR}=1.75$ (IC $95 \%=0.96-3.15)$. El resto de los RR están resumidos en la Tabla 2. Los RR están representados en la Figura 1.

Se compararon las medias de las FEVi0 y FEVi1 con prueba t de Student, en la que se encontró una 


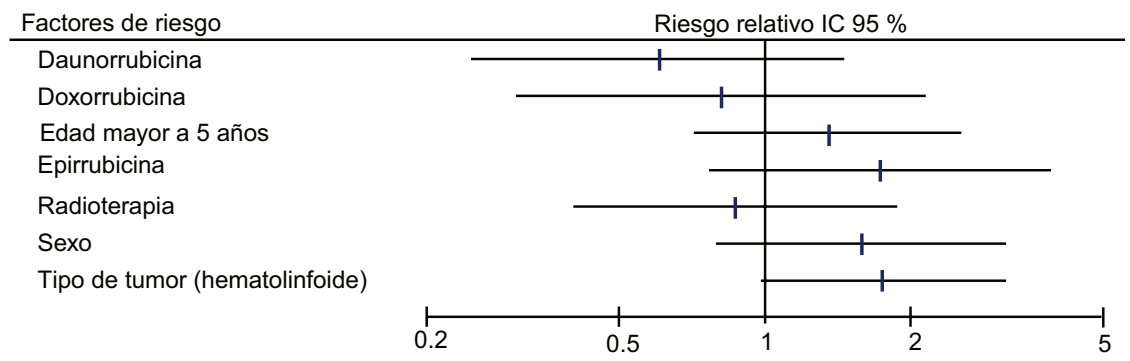

Figura 1. Principales factores de riesgo asociados al desarrollo de cardiotoxicidad.

\begin{tabular}{|c|c|c|c|c|}
\hline \multirow[t]{2}{*}{ Variable } & \multicolumn{2}{|c|}{ Cardiotoxicidad } & \multirow{2}{*}{$\begin{array}{l}\text { Riesgo } \\
\text { relativo }\end{array}$} & \multirow[t]{2}{*}{ IC $95 \%$} \\
\hline & $\begin{array}{c}\text { Sí } \\
(n=26)\end{array}$ & $\begin{array}{c}\text { No } \\
(n=39)\end{array}$ & & \\
\hline Masculino & 19 & 22 & 1.6 & $0.78-3.21$ \\
\hline Doxorrubicina versus & 3 & 6 & \multicolumn{2}{|c|}{ Referencia } \\
\hline Epirrubicina & 21 & 25 & 1.4 & $0.51-3.63$ \\
\hline Daunorrubicina & 4 & 11 & 0.81 & $0.22-2.78$ \\
\hline Tumor hematolinfoide & 15 & 12 & \multirow[t]{2}{*}{1.8} & \multirow[t]{2}{*}{$1.01-3.22$} \\
\hline Tumor sólido & 12 & 27 & & \\
\hline Edad $>5$ años & 16 & 18 & 1.4 & $0.72-2.57$ \\
\hline Radioterapia & 5 & 9 & 0.9 & $0.39-1.88$ \\
\hline
\end{tabular}

$\mathrm{RR}=$ riesgo relativo, IC $95 \%$ = intervalo de confianza de $95 \%$

diferencia de medias de $10 \%(p=0.001)$, que puede observarse en la Figura 2.

La prevalencia de la CTX en nuestra población fue de $60 \%$. El resto de las complicaciones asociadas a antraciclinas fueron neutropenia y fiebre (18\%), mucositis $(6.2 \%)$, trombocitopenia y sepsis (3.1\%).

Se calculó un modelo de regresión logística en el que la variable dependiente fue la CTX, ajustada por la edad y la dosis acumulada, las principales variables de confusión (Tabla 3).

Se utilizó el método de regresión logística por pasos descendentes para que en el modelo quedarán las principales variables asociadas al desarrollo de cardiotoxicidad. En el paso 4 del modelo, la variable epirrubicina tuvo una razón de momios $(\mathrm{RM})=4.69$ (IC $95 \%=0.75-29.03$ ), la dosis acumulada presentó una $\mathrm{RM}=1.004$ (IC $95 \%=1.001-1.007$ ).

Debido a que la dosis acumulada fue la única variable asociada a CTX, se realizó una curva ROC

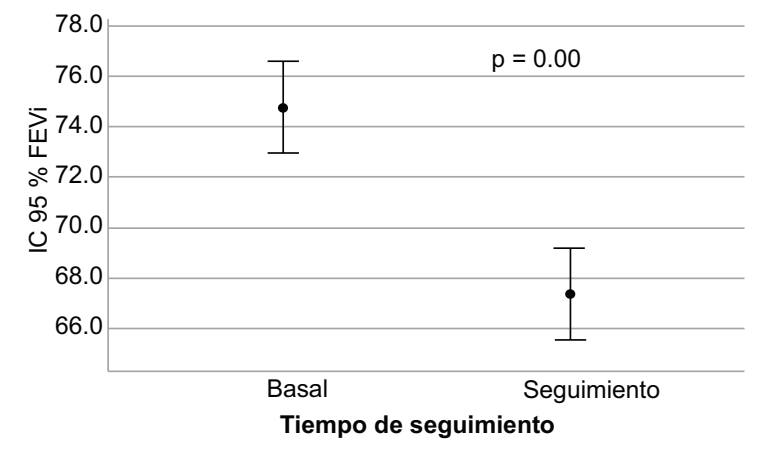

Figura 2. Comparación de las medias de fracción de expulsión basal versus fracción de expulsión a los 12 meses. FEVi = fracción de eyección.

(Figura 3), en la cual se encontró un área bajo la curva de 0.716 (IC $95 \%=0.55-0.882$ ). La dosis de $260 \mathrm{mg}$ tuvo una RVP de 1.84; la de $380 \mathrm{mg}$, de 2.15; y la de $430 \mathrm{mg}$, de 3.51 (Figura 4).

\section{Discusión}

Se han identificado algunos factores de riesgo asociados a la CTX por antraciclinas, los cuales son potencialmente modificables, como la dosis acumulada, la velocidad de aplicación, los tratamientos concomitantes o la actividad física. Sin embargo, existen pocos estudios con seguimiento a largo plazo en los que se ajusten estos posibles factores de riesgo de conformidad con los posibles factores confusores. Hasta donde es de nuestro conocimiento, este es el primer estudio en México en el que se evalúan los factores de riesgo con modelos multivariados para el desarrollo de CTX.

El presente estudio se centró en los pacientes supervivientes de cáncer, es decir, en quienes desarrollaron la CTX después del tratamiento, lo cual descarta a los pacientes que experimentaron toxicidad aguda, como lo describimos en otras investigaciones. ${ }^{17}$

Los reportes internacionales señalan que la prevalencia de la CTX en pacientes que reciben 
Tabla 3. Modelo de regresión logística para el desarrollo de cardiotoxicidad asociada al uso de antraciclinas

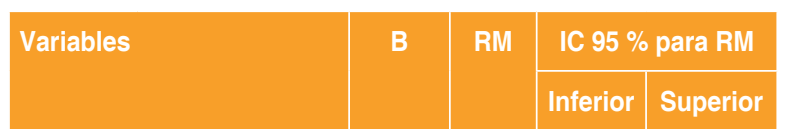

Paso $1(p=0.116)$ \begin{tabular}{|l|l|l|l|}
\hline 0.04 & 1.04 & 0.17 & 6.46 \\
\hline
\end{tabular}

\begin{tabular}{|l|l|l|l|l|}
\hline Epirrubicina & 1.93 & 6.88 & 0.81 & 58.4 \\
\hline
\end{tabular}

\begin{tabular}{|l|l|l|l|l|} 
Diagnóstico* $^{*} 0.379$ & 1.461 & 0.264 & 8.077 \\
\hline
\end{tabular}

\begin{tabular}{|l|l|l|l|l|}
\hline Edad $^{\star *}$ & -0.726 & 0.484 & 0.056 & 4.202 \\
\hline
\end{tabular}

\begin{tabular}{|l|l|l|l|l|}
\hline Dosis acumulada & 0.003 & 1.003 & 1 & 1.007
\end{tabular}

Paso $2(p=0.062)$

\begin{tabular}{|l|l|c|c|c|c|}
\hline$r^{2}=0.215$ & Epirrubicina & 1.926 & 6.859 & 0.815 & 57.709 \\
\hline Diagnóstico* & 0.38 & 1.462 & 0.265 & 8.083 \\
\hline Edad** & -0.704 & 0.494 & 0.071 & 3.427 \\
\hline Dosis acumulada & 0.003 & 1.003 & 1 & 1.007 \\
\hline
\end{tabular}

Paso $3(p=0.029)$

\begin{tabular}{|l|l|l|l|l|l|}
$r^{2}=0.213$ & Epirrubicina & 1.784 & 5.955 & 0.795 & 44.586
\end{tabular}

\begin{tabular}{|l|l|l|l|l|}
\hline Edad $^{* *}$ & -0.752 & 0.471 & 0.069 & 3.237 \\
\hline
\end{tabular}

\begin{tabular}{|l|l|l|l|l|}
\hline Dosis acumulada & 0.003 & 1.003 & 1 & 1.007
\end{tabular}

Paso $4(p=0.013)$

\begin{tabular}{ll|l|l|l|l|}
$r^{2}=0.203$ & Epirrubicina & 1.546 & 4.692 & 0.758 & 29.035
\end{tabular}

\begin{tabular}{|l|l|l|l|l|}
\hline Dosis acumulada & 0.004 & 1.004 & 1.001 & 1.007
\end{tabular}

*Diagnóstico hematolinfoide versus sólido. ${ }^{* \star} E$ dad $>5$ años.

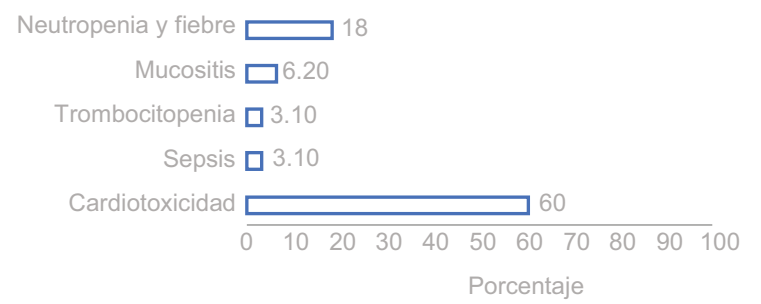

Figura 3. Prevalencia de las complicaciones asociadas a las antraciclinas.

antraciclinas es de aproximadamente $40 \%$; sin embargo, en nuestro análisis fue de $60 \%$, lo cual puede ser atribuido a que en nuestra población la incidencia de tumores hematolinfoides es mayor que en otras poblaciones.

En el modelo bivariado, el tumor hematolinfoide presentó un $R R=1.75$, que no fue significativo, probablemente por un tamaño de muestra insuficiente, ya que el límite inferior del intervalo de 0.97 indica una tendencia al riesgo. Se observó una situación similar con el sexo masculino y la epirrubicina; puede ser que estos factores sean significativos si se aumenta el tamaño de la muestra, como se indica en otros estudios. ${ }^{15}$

Si bien la evaluación cardiaca ha evolucionado, la reducción de la fracción de expulsión y la insuficiencia cardiaca congestiva sintomática (actuales desenlaces de la CTX $)^{18}$ generalmente ocurren después de semanas 0 años de finalizado el tratamiento. ${ }^{19} \mathrm{La}$ medición de la fracción de expulsión continúa empleándose en la práctica clínica por su fácil acceso y gran utilidad para determinar el inicio de los tratamientos de protección cardiaca. ${ }^{20}$

El mecanismo principal de la CTX inducida por las antraciclinas parece implicar la generación de radicales de oxigeno altamente reactivos, los cuales promueven la peroxidación lipídica que daña la membrana celular. ${ }^{21}$ Los efectos tóxicos de las antraciclinas en los miocardiocitos llevan al adelgazamiento de la pared del ventrículo izquierdo, lo que incrementa la poscarga y disminuye la contractilidad, origen de la insuficiencia cardiaca congestiva. ${ }^{22}$ Las guías europeas recomiendan la vigilancia de los pacientes con alto riesgo clínico, aun cuando no están bien descritos los factores de riesgo en los pacientes pediátricos. ${ }^{23}$

En la práctica clínica, el seguimiento de la remisión completa del cáncer es de un año después de terminar la quimioterapia, con lo cual se podría subestimar la incidencia de este fenómeno, en especial en los niños, con quienes se puede perder contacto.

En el modelo multivariado se realizó el ajuste de acuerdo con las principales variables confusoras: edad, sexo, tipo de tumor y dosis acumulada. El tamaño de la muestra limitó el número de variables en el modelo, sin embargo, en la dosis acumulada se obtuvo una $\mathrm{RM}=1.004$, que resultó ser estadísticamente significativa. Lo anterior tiene implicaciones importantes: al parecer es la dosis total y no el tipo de antraciclina la que se asocia a las complicaciones cardiacas en los niños, a diferencia de lo que ocurre en los adultos.

Podemos relacionar este hallazgo con el hecho de que en el modelo bivariado los tumores hematolinfoides fueron el factor de riesgo principal, debido a que los pacientes con este tipo de tumores reciben las dosis más altas de quimioterapia y por un periodo más largo.

La importancia de hacer estos análisis en cada país radica en las variantes genéticas que modifican la farmacología de la quimioterapia. ${ }^{24}$ 

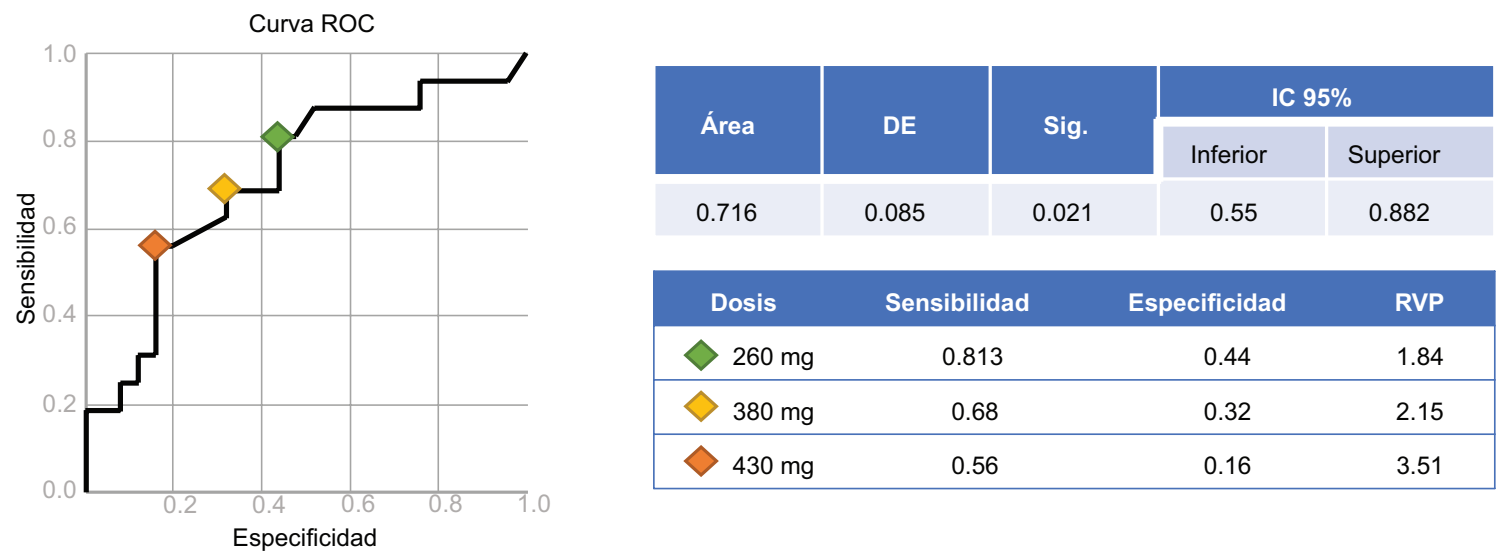

\begin{tabular}{|cccc|}
\hline Dosis & Sensibilidad & Especificidad & RVP \\
\hline $260 \mathrm{mg}$ & 0.813 & 0.44 & 1.84 \\
\hline $380 \mathrm{mg}$ & 0.68 & 0.32 & 2.15 \\
\hline $430 \mathrm{mg}$ & 0.56 & 0.16 & 3.51 \\
\hline
\end{tabular}

Figura 4. Curva ROC de la asociación de la dosis acumulada y la mayor verosimilitud con la cardiotoxicidad. RVP = razón de verosimilitud positiva. $D E$ = desviación estándar. Sig. = significación.

El estudio que presentamos es parte del seguimiento de una cohorte de supervivientes pediátricos de cáncer, el cual ha permitido detectar otros eventos adversos como pérdida auditiva, ${ }^{25}$ nefrotoxicidad, ${ }^{26,27}$ neutropenia y fiebre, ${ }^{28,29}$ de ahí la relevancia de los estudios de seguimiento ${ }^{30}$ y de los ensayos clínicos que permitan tratar de forma adecuada las consecuencias de la toxicidad por la quimioterapia. ${ }^{31}$ No basta con la curación, se requiere continuar con los esfuerzos para limitar los eventos adversos reversibles y no mortales y los irreversibles. ${ }^{32,33}$

En esta investigación proponemos que las dosis altas de las antraciclinas (sin importar su tipo) constituyen el principal factor de riesgo. De tal suerte, los clínicos podrán ofrecer tratamientos protectores a los niños que requieran dosis altas de antraciclinas, lo cual podrá, en teoría, disminuir una de las complicaciones más catastróficas.

\section{Conclusión}

El principal factor de riesgo asociado a la cardiotoxicidad temprana en niños mexicanos supervivientes de cáncer es la dosis acumulada mayor de $430 \mathrm{mg}$.

\section{Agradecimientos}

Este estudio forma parte de \#iniciativaM. Agradecemos a los padres de los niños que permitieron la realización del estudio.

\section{Conflicto de intereses}

Ninguno.

\section{Financiamiento}

La presente investigación no ha recibido ninguna beca específica de agencias de los sectores público, comercial, o sin ánimo de lucro.

\section{Responsabilidades éticas}

Protección de personas y animales. Los autores declaran que los procedimientos se realizaron de conformidad con las normas éticas del comité de experimentación humana responsable y de acuerdo con la Asociación Médica Mundial y la Declaración de Helsinki.

Confidencialidad de los datos. Los autores declaran que han seguido los protocolos de su centro de trabajo sobre la publicación de datos de pacientes.

Derecho a la privacidad y consentimiento informado. Los autores han obtenido el consentimiento informado de los pacientes referidos en el artículo, documento en poder del autor de correspondencia.

\section{Bibliografía}

1. Lipshultz SE, Colan SD, Gelber RD, Pérez-Atayde AR, Sallan SE, Sanders SP. Late cardiac effects of doxorubicin therapy for acute lymphoblastic leukemia in childhood. N Engl J Med. 1991;324:808-815.

2. Fajardo-Gutiérrez A, Rendón-Macías ME, Mejía-Aranguré JM. Epidemiología del cáncer en niños mexicanos. Rev Med Inst Mex Seguro Soc. 2011;49:S43-S70.

3. Steinherz LJ, Steinherz PG, Tan CT, Heller G, Murphy ML. Cardiac toxicity 4 to 20 years after completing anthracycline therapy. JAMA. 1991;266:1672-1677.

4. 58a Asamblea Mundial de la Salud: Prevención y Control del Cáncer. Suiza: Organización Mundial de la Salud; 2015.

5. Instituto Nacional de Estadística y Geografía. Conteo de Población y Vivienda 2010. México: INEGl; 2011.

6. Rivas-Ruiz R, Villasís-Keever M, Miranda-Novales G, Castelán-Martínez $\mathrm{OD}$, Rivas-Contreras S. Outpatient treatment for people with cancer who develop a low-risk febrile neutropaenic event. Cochrane Database Syst Rev. 2019:3:CD009031.

7. Weiss RB. The anthracyclines: will we ever find a better doxorubicin? Semin Oncol. 1992;19:670-686. 
8. Mertens AC, Yasui Y, Neglia JP, Potter JD, Nesbit ME Jr, Ruccione K, Smithson WA, Robison LL. Late mortality experience in five-year survivors of childhood and adolescent cancer: The Childhood Cancer Survivor Study. J Clin Oncol. 2001;19:3163-3172.

9. Oeffinger KC, Mertens AC, Sklar CA, Kawashima T, Hudson MM, Meadows AT, et al. Chronic health conditions in adult survivors of childhood cancer. N Engl J Med. 2006;355:1572-1582.

10. Reulen RC, Winter DL, Frobisher C, Lancashire ER, Stiller CA Jenney ME, Skinner R, et al. Long-term cause-specific mortality among survivors of childhood cancer. JAMA. 2010;304:172-179.

11. Steinherz LJ, Steinherz PG, Tan CT, Heller G, Murphy ML. Cardiac toxicity 4 to 20 years after completing anthracycline therapy. JAMA 1991;266:1672-1677.

12. Kremer LC, van Dalen EC, Offringa M, Voûte PA. Frequency and risk factors of anthracycline-induced clinical heart failure in children: a systematic review. Ann Oncol. 2002;13:503-512.

13. McEvoy GK, editor. AHFS 2005 Drug Information. EE. UU.: American Society of Health-System Pharmacists; 2005. p. 972-982.

14. Repchinsky C. Compendium of pharmaceuticals and specialties. Canadá: Canadian Pharmacists Association; 2005.

15. McGowan JV, Chung R, Maulik A, Piotrowska I, Walker JM, Yellon DM. Anthracycline chemotherapy and cardiotoxicity. Cardiovasc Drugs Ther. 2017;31:63-75.

16. Pérez-Rodríguez M, Palacios-Cruz L, Rivas-Ruiz R, Talavera JO. Clinical research XXIV. From clinical judgment to ethics in research on humans. Rev Med Inst Mex Seguro Soc. 2014:52:666-672.

17. Vargas-Neri JL, Castelán-Martínez OD, Estrada-Loza MJ, Betanzos-Cabrera Y, Rivas-Ruiz R. Anthracycline-induced cardiotoxicity: report of fatal cases. Rev Med Inst Mex Seguro Soc. 2016;54:404-408.

18. Chow EJ, Leger KJ, Bhatt NS, Mulrooney DA, Ross CJ, Aggarwal S, et al. Pediatric cardio-oncology: epidemiology, screening, prevention, and treatment. Cardiovasc Res. 2019;115:922-934.

19. Doxorubicin Product Monograph. Canadá: Mayne Pharma; 2002.

20. Henriksen PA. Anthracycline cardiotoxicity: an update on mechanisms, monitoring and prevention. Heart. 2018;104:971-977.

21. Gewirtz DA. A critical evaluation of the mechanisms of action proposed for the antitumor effects of the anthracycline antibiotics adriamycin and daunorubicin. Biochem Pharmacol. 1999;57:727-741.
22. Wouters KA, Kremer LC, Miller TL, Herman EH, Lipshultz SE. Protecting against anthracycline-induced myocardial damage: a review of the most promising strategies. Br J Haematol. 2005;131:561-578.

23. Bonadonna G, Monfardini S. Cardiac toxicity of daunorubicin. Lancet. 1969;1:837.

24. Zazuli Z, Vijverberg S, Slob E, Liu G, Carleton B, Veltman J, et al. Genetic variations and cisplatin nephrotoxicity: a systematic review. Front Pharmacol. 2018:9:1111.

25. Castelán-Martínez OD, Jiménez-Méndez R, Rodríguez-Islas F, Fierro-Evans M, Vázquez-Gómez BE, Medina-Sansón A, et al. Hearing loss in Mexican children treated with cisplatin. Int J Pediatr Otorhinolaryngol. 2014;78:1456-1460.

26. Jiménez-Triana CA, Castelán-Martínez OD, Rivas-Ruiz R, Jiménez-Mén$\operatorname{dez} R$, Medina A, Clark $P$, et al. Cisplatin nephrotoxicity and longitudinal growth in children with solid tumors: a retrospective cohort study. Medicine (Baltimore). 2015;94:e1413.

27. Gavrilovici C, Luca A, Antoniu AS, Gallaby K, Starcea M, Miron I, et al. How nephrotoxic is the cancer therapy in children? Farmacia. 2018;66:197-208

28. Castelán-Martínez OD, Rodríguez-Islas F, Vargas-Neri JL, Palomo-Colli MA, López-Aguilar E, Clark P, et al. Risk factors for febrile neutropenia in children with solid tumors treated with cisplatin-based chemotherapy. J Pediatr Hematol Oncol. 2016;38:191-196.

29. Gil-Veloz M, Pacheco-Rosas DO, Solórzano-Santos F, Villasís-Keever MA, Betanzos-Cabrera Y, Miranda-Novales G. Early discharge of pediatric patients with cancer, fever, and neutropenia with low-risk of systemic infection. Bold Med Hosp Infant Mex. 2018;75:352-357.

30. Zhou L, Rupa AP. Categorization and association analysis of risk factors for adverse drug events. EurL J Clin Pharmacol. 2018;74:389-404.

31. Pacheco-Rosas DO, Peregrino-Bejarano L, López-Aguilar JE, JuanShum L, Miranda-Novales MG. Piperacillin/tazobactam plus amikacin vs. piperacilin/tazobactam: treatment for children with febrile neutropenia. Rev Med Inst Mex Seguro Soc. 2019;57:65-73.

32. Cortés-Cerda R, Mora-Ríos FG, Insunza-Ramírez A, Mejía-Rohenes LC, Ruiz-Alva SK, Pérez García CK. Benign tumors that cause fractures in children. Acta Ortop Mex. 2018;32:283-286.

33. Ohtani K, Ide T, Hiasa KI, Sakamoto I, Yamashita N, Kubo M, et al. Cardioprotective effect of renin-angiotensin inhibitors and $\beta$-blockers in trastuzumab-related cardiotoxicity. Clin Res Cardiol. 2019;108:1128-1139. 